\title{
Design of an Electric Road-Rail Vehicle
}

\author{
Xu shun ${ }^{1}$, Hou xiaobao ${ }^{1}$, Bing Chenyang ${ }^{1}$, Ma Zetao $^{2}$ and Cui Shumei ${ }^{2}$ \\ ${ }^{1}$ Engineering and Technology Department, CRRC, Qingdao, China. \\ ${ }^{2}$ Institute of Electromagnetic and Electronic Technology, Harbin Institute of Technology, Harbin, China.
}

\begin{abstract}
In this paper, an electric road-rail vehicle and its control system are designed. The electric vehicle equipped a train couplers in front of it is used as a movable electric signal test bench for China Rail High-speed (CRH). Firstly, architecture of the vehicle is introduced. In the powertrain, hydraulic system is designed for the transition from road mode to rail mode, and vice versa. And in both road and rail modes, the vehicle is driven by an induction machine. Secondly, to improve the driving performance and satisfy different driving conditions, particular control strategy for the induction machine is put forward by using a load torque observer based on sliding mode control theory. The load observer based induction machine control system can improve the speed stability and robustness to the unexpected load disturbing. Finally, experiment is done and the results verify that the algorithm works well.
\end{abstract}

\section{Introduction}

Road-rail vehicle ${ }^{[1,2]}$ is used as a train-trailer or for freight transport on rail. According to its different driving requirement, the vehicle should be able to run on the road as well as the rail. Because of the environmental pollution and the energy crisis, nowadays manufacturers and governments pay more and more attention to clean energy vehicles ${ }^{[3,4,5]}$.As the development of the energy storage systems, long distance and heavy load condition is now not a problem anymore for electric vehicle. Due to the significant advantage of electric vehicle, in this paper, we propose a kind of electric road-rail vehicle which uses both hydraulic system and pure electric drive system. The electric road-rail vehicle is used as a test bed in workshop for automatic test of train couplers electric signals. The electrification leads to no pollution and noise, which would significantly improve the comfort of the operating environment.

In the paper, the architecture and control structure of the road-rail electric vehicle is presented and the control system is designed. The hydraulic system for the mode transition is introduced. Then the electric driving system and the control strategy is designed. To improve the driving performance, a load torque observer based on sliding mode control is design to realize robust speed control of the induction machine driving system. Simulation is done and the results show good performance of the control method.

\section{Architecture of the proposed electric Road-rail vehicle}

The electric road-rail vehicle is designed as a movable test bed for $\mathrm{CRH} 2$ train couplers. It is used to test the electrical signals of the train couplers for fault diagnosis. To efficiently fulfill the experiments, which means less operators and faster operation, the vehicle should be designed properly. Firstly, the vehicle should be driven as a normal vehicle on the road.Secondly, when testing the train couplers, the vehicle should be driven on the rail to couple with the under testing coupler on the train, which requires the speed of the vehicle should be robustly controlled. Furthermore, when running on the rail, the operators should be able to drive the vehicle remotely, which means the vehicle should be controlled by using a remote control.

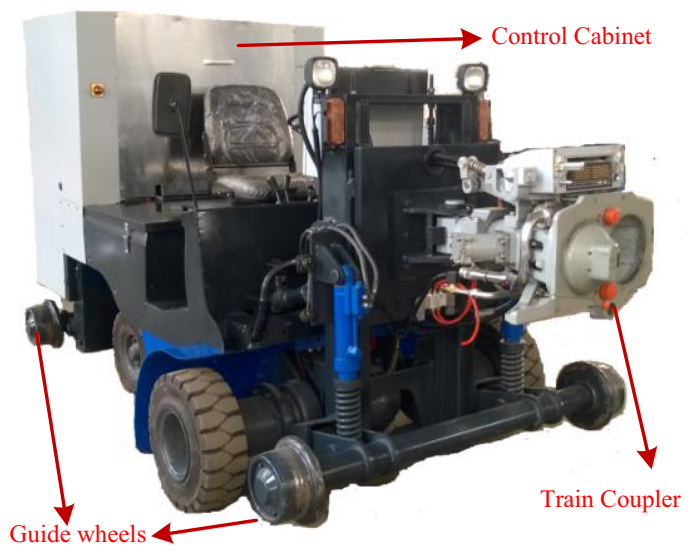

Figure 1. The electric road-rail vehicle

As shown in Figure 1, the specific designed electric vehicle equipped a train coupler in front and the control cabinet in rear. The control cabinet includes a test control system and a driving system. The test control system is 
used for the coupler signals testing and the driving control system is used for driving control.

\subsection{Architecture of the proposed electric road- rail vehicle}

In the electric road-rail vehicle, both hydraulic system and electric driving system is required to satisfy different driving mode. The hydraulic system is designed to realize the mode transition. For example, when the vehicle is required to run on the rail, the guide wheels should be put down to engage the rail for direction guidance, and vice versa. The vehicle is propelled by an induction machine, which is driven by vector controlled inverter. The Architecture is shown in Fig. 3.

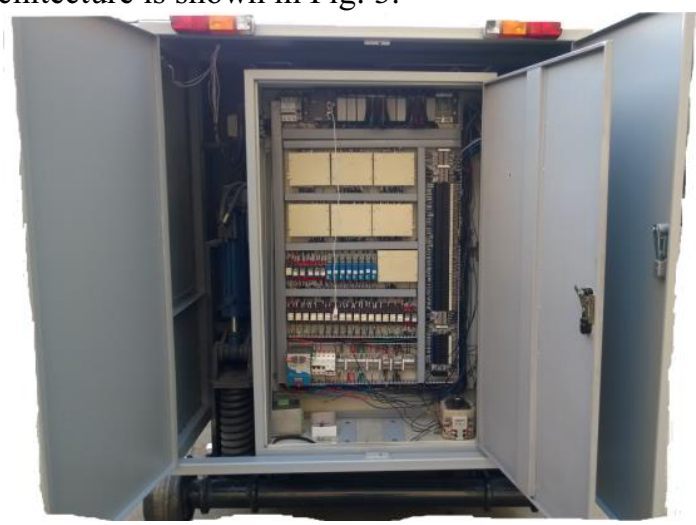

Figure 2. The test control system for train couplers

In the electric road-rail vehicle, the induction machine is used to propel the vehicle both in road mode and rail mode. PLC is used as the center controller and receives instruction from the driver. Guide wheels are used to guide the vehicle along the rail when the vehicle runs on the rail. When the guide wheels are put down, the rear of the vehicle is lifted up to disable the steering system.

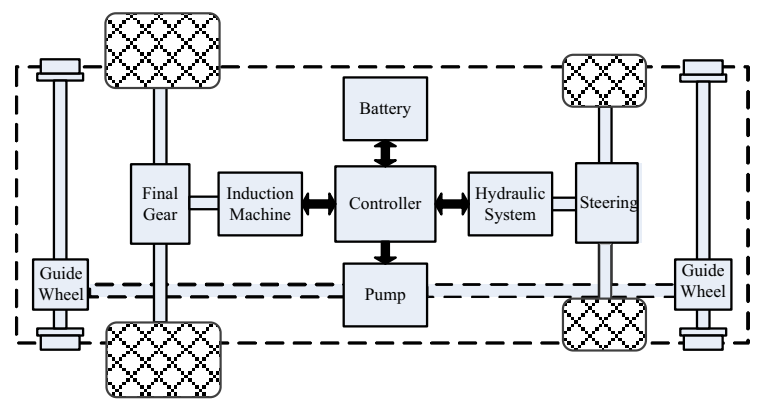

Figure 3. Architecture of the electric road-rail vehicle

Use a two-column format, and set the spacing between the columns at $8 \mathrm{~mm}$. Insert "MATEC Web of Conferences" in even headers and the short form title of the conference in odd headers, except the first one. Please check with the organiser the exact short title of the conference. Do not add any page numbers. The articles will be quoted as follows:

\subsection{Hydraulic system design of the road-rail vehicle}

The hydraulic system is designed for the steering system and the guide wheels control system.
In the guide wheel control system, electromagnetic valves are used to realize the lifting and descending of the guide wheels. When the vehicle runs on the rail, only the guide wheels in rear and the tires in front brace the vehicle, while the guide wheels in front only guides the vehicle and the tires in rear is lifted away from the ground. This simplified designation guarantees that when the vehicle runs on the rail, the steering system is disabled, which means the vehicle is restricted along the rail direction reliably.

The highest pressure of the hydraulic system is $17 \mathrm{Mpa}$ which is enough for the action of the guide wheels in rear.

Besides, the hydraulic system makes sure that the vehicle is able to turn around in nearly 360 degree for free turning in the factory.

\subsection{Induction machine drive system}

In the electric road-rail vehicle, the induction machine is used. In different driving mode, the induction machine should be controlled in different ways ${ }^{[6,7,8]}$.

When the vehicle runs on the road, the center controller receives torque command from the driver, which means the induction machine should be defined as torque controlled. In the torque control mode, the vehicle can be driven like it is a traditional vehicle. While when the vehicle runs on the rail, in some occasion, high performance of speed control is required for specific usage. For example, when the vehicle is required to be remotely controlled and located precisely. Thus, in the induction machine drive system, torque control mode and speed control mode is designed respectively to satisfy different driving acquirement.

In both torque control mode and speed control mode, the induction machine is controlled by a voltage fed inverter, applying vector control algorithm. While in torque control mode, the speed closed loop is canceled.

The diagrams of the two control strategies are shown in Fig. 4 and Fig. 5.

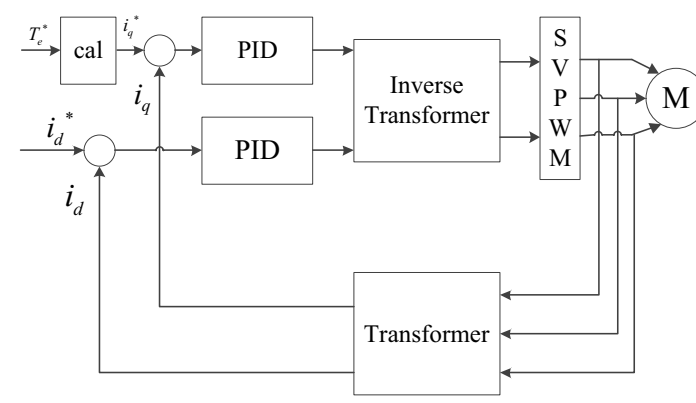

Figure 4. Torque control diagram of the induction machine

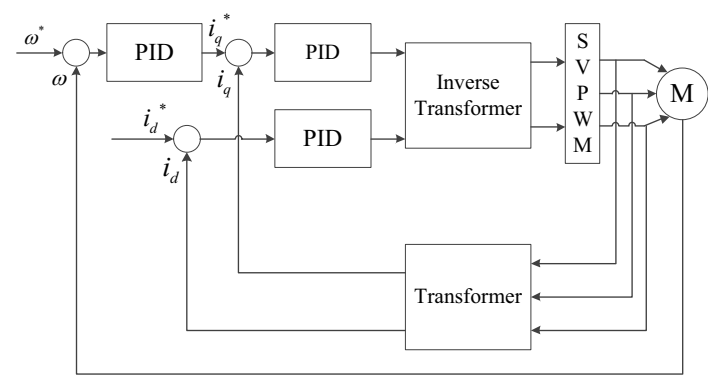

Figure 5. Speed control diagram of the induction machine 
In the torque control mode, only current closed loop is required to realize torque control. While in speed control mode, the controller should include both current closed loop and speed closed loop. Due to the particular driving condition of the vehicle, field weakening is not an important point of the control strategy of the electric machine. Thus, no field weakening control strategy is discussed in this paper.

\section{Load Torque Observer based on sliding mode theory}

When the electric road-rail vehicle runs on the rail, high performance of speed control is required for particular application. To improve the robust performance of the speed control, load torque feed forward control strategy is used in the IM drive system.

\subsection{Induction machine model}

The induction machine model in the dq frame can be described as:

$\left[\begin{array}{l}u_{d s} \\ u_{d s} \\ 0 \\ 0\end{array}\right]=\left[\begin{array}{cccc}R_{s}+p L_{s} & -\omega_{s} L_{s} & p L_{m} & -\omega_{s} L_{m} \\ \omega_{s} L_{s} & R_{s}+p L_{s} & \omega_{s} L_{m} & p L_{m} \\ p L_{m} & -\Delta \omega_{r} L_{m} & R_{r}+p L_{r} & -\Delta \omega_{r} L_{r} \\ -\Delta \omega_{r} L_{m} & p L_{m} & \Delta \omega_{r} L_{r} & R_{r}+p L_{r}\end{array}\right]\left[\begin{array}{l}i_{d s} \\ i_{q s} \\ i_{d r} \\ i_{q r}\end{array}\right](1)$

Where $u_{d s}$ and $u_{d s}$ are the stator voltage in dq frame respectively and $i_{d s} i_{q s}$ are the stator current respectively.

$R_{s}$ is the stator resistance and $R_{r}$ is the rotor resistance.

$L_{s}$ is the stator inductance and $L_{r}$ is the rotor induction. $L_{m}$ is the mutual inductance.

Then, the electro-magnetic torque of the IM machine can be expressed as:

$$
T_{e}=p_{m} L_{m}\left(i_{d s} i_{q r}-i_{q s} i_{d r}\right)
$$

Where $T_{e}$ is the electro-magnetic torque and $p_{m}$ is the pole-pair number.

When implement vector control strategy, the inductance machine speed control system is shown in figure5. Current and speed information is used for double closed loop control to improve the responsibility and control performance of the driving system.

\subsection{Load observer based on sliding mode theory}

Sliding mode control has been applied in induction machine and PMSM machine control widely. For example, different kind of torque observers and sensorless control strategies use sliding mode control theory to improve the control performance of the machine [9-15].

The motion equation of the induction machine can be described as:

$$
\frac{d \omega}{d t}=\frac{1}{J} T_{e}-\frac{1}{J} T_{l}
$$

Where $\omega$ is the rotating speed, $J$ is the equivalent inertia and $T_{l}$ is the load torque.
To apply the sliding mode theory in a load torque observer, the state equation is built:

$$
\frac{d \hat{\omega}}{d t}=\frac{1}{J} T_{e}-k \operatorname{sgn}(\hat{\omega}-\omega)
$$

Where $\hat{\omega}$ is the estimated rotor speed, and sgn is the signal function.

Combining the equation (3) and equation (4), the speed error is now expressed as:

$$
\frac{d \tilde{\omega}}{d t}=\frac{1}{J} T_{l}-k \operatorname{sgn}(\tilde{\omega})
$$

Where $\tilde{\omega}$ is the speed error between the estimated speed and the real rotor speed.

According to equation (5), when the speed error equals to zero, then the load torque can be expressed as:

$$
T_{l}=J k \operatorname{sgn}(\tilde{\omega})
$$

To stabilize the control loop, the Lyapunov stabilization condition is constructed as:

$$
\left(\frac{1}{2} s^{2}\right)^{\prime}=s \dot{s} \leq 0
$$

Combining equation (6) and equation(7), the stabilization equation is now described as:

$$
\tilde{\omega} \dot{\tilde{\omega}}=\frac{\tilde{\omega}}{J} T_{l}-k \tilde{\omega} \operatorname{sgn}(\tilde{\omega}) \leq 0
$$

Thus, the relation below should be satisfied:

$$
k \geq\left|\frac{T_{l}}{J}\right|
$$

Furthermore, to acquire smooth load torque signal, a first order filter is used to filter the high frequency chattering of the observed signal:

$$
G(s)=\frac{\omega_{0}}{s+\omega_{0}}
$$

Where $\omega_{0}$ is the cut-off frequency.

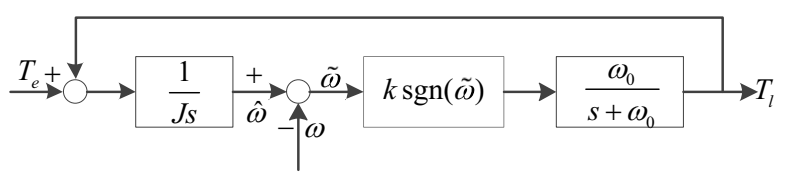

Figure 6 Load torque observer based on sliding mode theory

The diagram of the load torque observer based on sliding mode theory is shown in Figure 6.

\subsection{Feed forward Torque Control Strategy}

In the electric road-rail vehicle, the vector controlled strategy is investigated. To acquire high performance of speed control, the load torque feed forward control is implemented. The load torque is added into the control loop to improve the responsibility of the control system. When the driving conditions of the vehicle changed, the vehicle speed would change at the same time, the sliding mode observer then calculates the dynamic load torque and feeds to the current closed loop, which means the dynamic tuning process of the current closed loop is significantly cut short thus the responsibility and the robustness of the driving system is improved.

The complete control diagram of the induction machine is shown in fig7.The load torque observer 
calculates the load torque and then the load torque is fed forward to the control loop to improve the robust performance.

The control strategy is implemented in a digital processor. As shown in figure8 and figure9, the real speed follows the target speed well. In figure9, disturbance is added at 50 second, the experiment results shows that the driving system has a little oscillation. This is because the sliding mode observer has chattering characteristic.

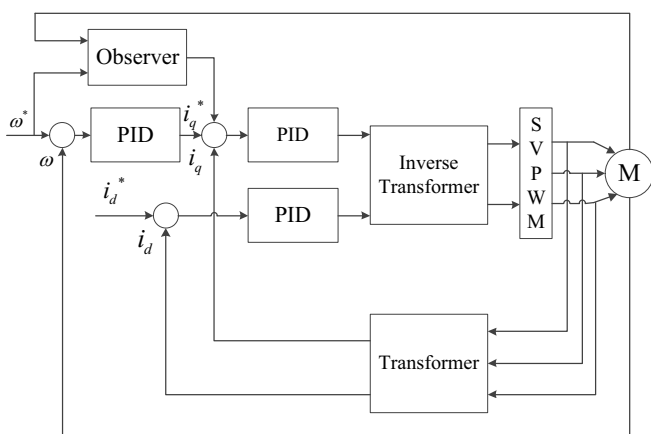

Figure 7. Feed forward speed control diagram of the induction machine

The control strategy is implemented in a digital processor. As shown in Figure 8 and Figure 9, the real speed follows the target speed well. In figure9, disturbance is added at 50 second, the experiment results shows that the driving system has a little oscillation. This is because the sliding mode observer has chattering characteristic.

\section{Experimental results}

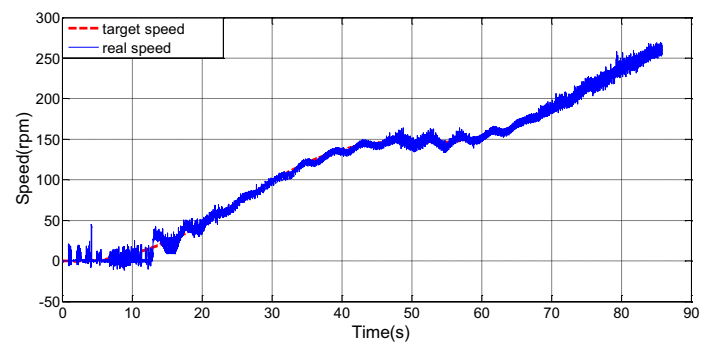

Figure 8. The speed performance of the vehicle

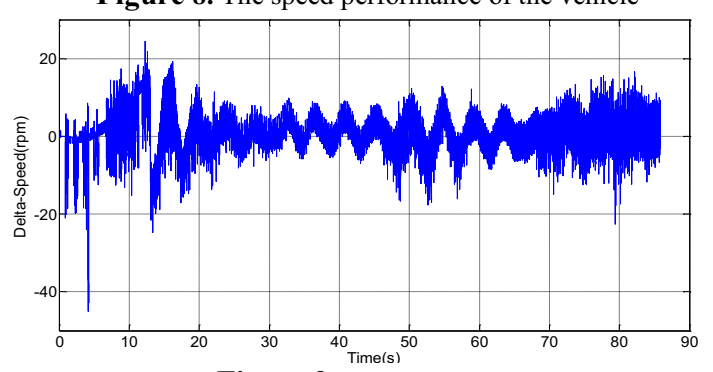

Figure 9. The speed error

\section{Conclusion}

In this paper, we design the control system for an electrified road-rail vehicle. In the vehicle, for different usage, the hydraulic system is designed to fulfill transition from road vehicle to rail vehicle, and vice versa. In the other hand, different induction machine control strategies are used for specific applications. That is, torque control is used for road driving and speed control is used for rail driving. A sliding mode torque observer is designed to improve the speed control performance of the vehicle. Simulation is done to verify the correctness of the algorithm.

\section{References}

1 Pei Lei, "Research on control system of electric road-rail towing vehicle", Harbin Institute of Technology, 2010.

2 Road to Rail. SRS

3 C. C. Chan. The State of the Art Electric and Hybrid Vehicles. Proceedings of The IEEE. 2002, 90(2):247 275

4 C. C. Chan. Electric Vehicles: Charge Forward. IEEE Power and Energy Magazine. 2004:2 12

59 Skudelny, Hans Christoph. Modern Directions of Research on Drive Systems for Electric Rail and Road Vehicles. 1980, 71(10):280 285.

6 Peter Vas. Vector control of AC Machines [M]. NewYork: Oxford University Press, 1990.

7 Peter Vas. Sensorless Vector and Direct Torque Control [M]. NewYork: Oxford University Press, 1998.

8 Vas P, Willems J L, Brown J E. The Application of Space-phasor Theory to the Analysis of Electrical Machines with Space Harmonics[J]. Archiv fur Elektrotechnik, 1987, 69:359-363.

9 Zhang Xiaoguang, Sun Li, Zhao Ke, "Sliding Mode Control of PMSM Based on a Novel Load Torque Sliding Mode Observer", Proceedings of the CSEE, 2012

10 Lai C K, Shyu K K. A novel motor drive design forincremental motion system via sliding-mode controlmethod $[\mathrm{J}]$. IEEE Transaction on Industrial Electronics, 2005, 52(2): 499-507.

11 Roy A M C, Mohammad S I. Application of a sliding mode observer for position and speed estimation in switched reluctance motor drives[J]. IEEE Transactions onIndustry Application, 2001, 37(1): 51-58.

12 Tong Kewen, Zhang Xing. Sliding mode variable structure control of permanent magnet synchronous based on a novel reaching law $[\mathrm{J}]$. Proceedings of the CSEE, 2008, 28(21): 102-106(in Chinese).

13 Roy A M C, Mohammad S I. Application of a sliding mode observer for position and speed estimation in switched reluctance motor drives $[\mathrm{J}]$. IEEE Transactions on Industry Application, 2001, 37(1): 51-58.

14 Wai R. Total sliding-mode controller for PM synchronous servo motor drive using recurrent fuzzy neural network [J]. IEEE Transactions on Industrial Electronics, 2001, 48(5): 926-944.

15 Ha Q P, Nguyen Q H, Rye D C, et al. Fuzzy slidingmode controllers with applications [J]. IEEE Transactions on Industrial Electronics, 2001, 48(1): $38-41$. 